\title{
Empirical Model for the Transconductance-Current Dependence of Short-Channel MOSFETs
}

\author{
Vladimir I. Prodanov
}

\begin{abstract}
I dependence of sub-micrometer MOSFETs. The transconductance-current expression given by the Advanced Compact Model (ACM) is reviewed and simple modification is proposed. The modification yields an expression which (with proper parametrization) captures the $g_{m}-I$ dependence of short-channel MOSFETs. The proposed expression is "universal" in the sense that it is capable of modeling the $g_{\mathrm{m}}-\mathrm{I}$ dependence of long-channel MOSFETs, short-channel MOSFETs, and resistively-degenerated BJTs.
\end{abstract}

\section{INTRODUCTION}

Transconductance, commonly denoted $g_{m}$, is the slope of the transfer characteristic of a transistor evaluated at the "operating point". For a MOSFET, this is:

$$
\left.g_{m} \equiv \frac{d i_{D}}{d v_{G S}}\right|_{o . p .}
$$

The importance of the device transconductance is best understood in regard to an amplifier design. Here we will state several related facts that are very well-known.

1. The voltage gain of single-transistor amplifier cannot exceed the product $g_{m} R_{\text {Load }}$.

2. The mean-squared thermal noise of transistors can be written in terms of their $g_{m}$ [1]. Therefore, the value of the device transconductance has direct impact upon the noise performance of the circuits implemented.

3. The input linear range of a Common-Source amplifier with no source degeneration is approximately $40 \%$ of the $I_{D} / g_{m}$ ratio. Similar "rule" applies to CommonEmitter amplifiers: BJT-dictated input linear range is approximately $20 \%$ of $I_{C} / g_{m},[2]$.

4. Strong correlation exists between the transconductance value of the device and the power dissipation of the circuit (for Class-A circuits). This is related to the fact that the bias current determines both the $g_{m}$ of the device and the DC power consumption of the circuit.

The last two facts suggest that the $\mathrm{g}_{\mathrm{m}} \mathrm{I}$ dependence of a transistor has an important role in the design of analog circuits. Indeed, there are many publications that teach optimization of CMOS circuits based upon transistor $g_{m} / I$ ratio [3]-[7]. Most of these publications make use the Advanced Compact MOS Model. The $\mathrm{g}_{\mathrm{m}}-\mathrm{I}$ relation of a MOSFET in saturation, according to the ACM [3], [8] is:

$$
g_{m} \approx \frac{2}{1+\sqrt{1+I_{D} / I_{S}}} \times \frac{I_{D}}{n U_{T}}
$$

The normalization current, $I_{S}$, is defined as follows:

$$
I_{S} \equiv \frac{1}{2} n \mu C_{o x} \frac{W}{L} U_{T}^{2}
$$

$U_{T}$ in (2) and (3) denotes the thermal voltage $k T / q$ and $n$ is the so-called "slope factor" - a dimensionless quantity with value ranging from 1 to 2 . More information regarding the slope factor and the origin of expression (2) can be found elsewhere [3], [8]. The rest of the parameters in (3) have their usual meaning.

The ratio of the bias current $I_{D}$ to the normalization current $I_{S}$ determines the "inversion level" of the MOS channel. When $I_{D}$ is much smaller than $I_{S}$, the device is in weak inversion and its gate transconductance is proportional to the bias current, $g_{m} \approx I_{D} /\left(n U_{T}\right)$; the $g_{\mathrm{m}}$-I relation resembles that of a BJT. When $I_{D}$ is much larger than $I_{S}$, the device is said to operate in strong inversion. The transconductance of a "longchannel" MOSFET in strong inversion is proportional to the square-root of the bias current. This is consistent with the Shichman-Hodges (Spice Level 1) MOSFET model [9].

Expression (2) is relatively simple and in theory, valid in weak, moderate and strong inversion. This makes it useful for design of analog circuits. The shortcoming of the ACM $\mathrm{g}_{\mathrm{m}}$-I expression is that it fails to capture the $\mathrm{g}_{\mathrm{m}}$-I relation of "short-channel" MOSFETs operated in strong inversion. This shortcoming is related to the fact that the model does not account for the reduction of the carrier mobility with lateral field. A brute-force attempt to include such dependence will complicate the model, diminishing significantly its usefulness for circuit design.

An alternative strategy for improving the accuracy of the model while preserving its simplicity is presented here. The approach is based upon two important observations. Those are discussed in Section II. 


\section{ONE Function, Two Distinctly-DifFERENT CASES}

The "long-channel" MOSFET model (2) belongs to the following general class of functions:

$$
g_{m}=\frac{2}{1+\left(1+I / I_{\text {norm }}\right)^{m}} \times \frac{I}{V_{\text {norm }}}
$$

Here $V_{\text {norm }}$ and $I_{\text {norm }}$ are "normalization" quantities. They ensure the correctness of the transconductance dimensions (Ampere/Volt).

The impact of the dimension-less exponent $m$ is best understood by examining the behavior of expression (4) for extreme values of the bias current. When $I<<I_{\text {norm }}$, expression (4) reduces to $g_{m}=I / V_{\text {norm }}$. This means that $m$ has no impact upon the $\mathrm{g}_{\mathrm{m}}$-I behavior for small values of $I$. When $I>>I_{\text {norm }}$, the transconductance becomes proportional to $I^{I-m}$. If the exponent $m$ has value $1 / 2$, the $g_{m}$ will have square-root dependence upon the bias current. If $m$ is 1 , the $g_{m}$ will not be a function of the bias current. Increasing the value of $m$ from $1 / 2$ to 1 "flattens" the $g m-I$ curve. This is illustrated in Fig. 1. Also illustrated, is that making $m$ larger than unity leads to a curve with a non-monotonic behavior.

In the following we will show that the $g m-I$ expression of a resistively-degenerated $\mathrm{BJT}$ also belongs to the same family of functions. Hence, expression (4) is not specific to a "longchannel" MOSFET.

The effective transconductance of a resistively degenerated BJT, see Fig. 2, can formally be defined as:

$$
\left.g_{m(e f f)} \equiv \frac{d i_{C}}{d v_{B E^{\prime}}}\right|_{i_{C}=I}
$$

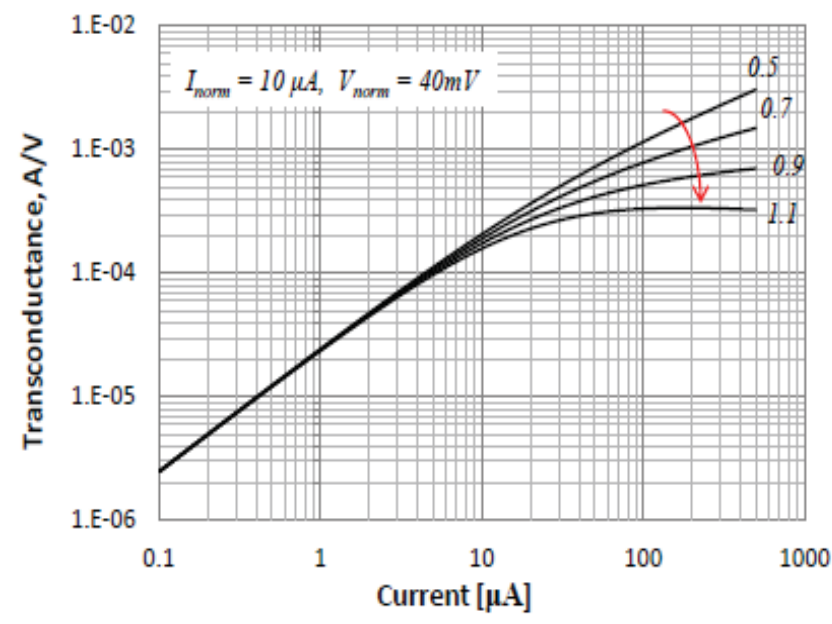

Figure 1. A $\log -\log$ plot of a family of $g_{m}-I$ curves with fixed $I_{\text {norm }}$, fixed $V_{\text {norm }}$ and $m$ varied from 0.5 to 1.1 in steps of 0.2 .

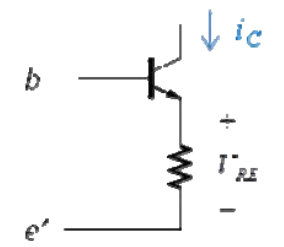

Figure 2. Schematic of an emitter-degenerated Bipolar Junction Transistor

Using "small-signal" analysis and/or the concept of feedback and loop gain, the $g_{m(e f f)}$ can be expressed in terms of the collector bias current $I$ and the voltage drop $V_{R E}$ as follows:

$$
g_{m(e f f)}=\frac{I}{U_{T}+V_{R E}}
$$

Expression (6) can also be rewritten as:

$$
g_{m(e f f)}=\frac{2}{1+\left(1+I / I_{\text {norm }}\right)^{1}} \times \frac{I}{U_{T}}
$$

Expression (7) clearly shows that the $g m-I$ dependence of Fig. 2 circuit is of the general form (4) where $m$ has value 1 .

The normalization current here is given by $I_{\text {norm }}=V_{T} /\left(2 \alpha R_{E}\right)$ where $\alpha$ is the common-base current gain of the BJT.

One wonders whether the $\mathrm{g}_{\mathrm{m}}$-I relation of a short-channel MOSFET is also member of the same family of functions. In Section III we argue that this is indeed the case. We show that it is possible to find a set of values for $I_{\text {norm }}, V_{\text {norm }}$ and $m$ to accurately curve-fit the $g_{m}-I$ dependence of sub-micrometer MOSFETs.

\section{CURVE-FITTING OF SPICE-DERIVED DATA}

The $g_{m}-I$ dependence of N-channel and P-channel devices from 6 different sub-micrometer CMOS processes were extracted using Spice. The transconductance was obtained by performing "operating point" analysis upon transistors connected in a "diode" configuration. Diode connection was used because it ensures active mode of operation irrespective of the value of the bias current and allows for a comparison of processes with different nominal supply voltages.

All six processes are available via MOSIS [10] and have feature size of $0.35 \mu \mathrm{m}, 0.25 \mu \mathrm{m}$ and $180 \mathrm{~nm}$. The $\mathrm{W} / \mathrm{L}$ of the devices studied is 10 and the drawn length $\mathrm{L}$ is the minimum one allowed by the respective technology. BSIM3v3 (HSPICE Level-49) models, found on MOSIS parametric datasheets [11], were used. Data were curve-fitted by adjusting $I_{\text {norm }}, V_{\text {norm }}$ and $m$ to minimize the maximum percent error.

A maximum error of less than $6 \%$ was achieved in all but one of the cases. Exemplary, Spice-extracted data and their corresponding curve-fitting functions are presented in Fig. 3, Fig. 4 and Fig. 5. The results for all cases considered here are summarized in Table I and Table II. 

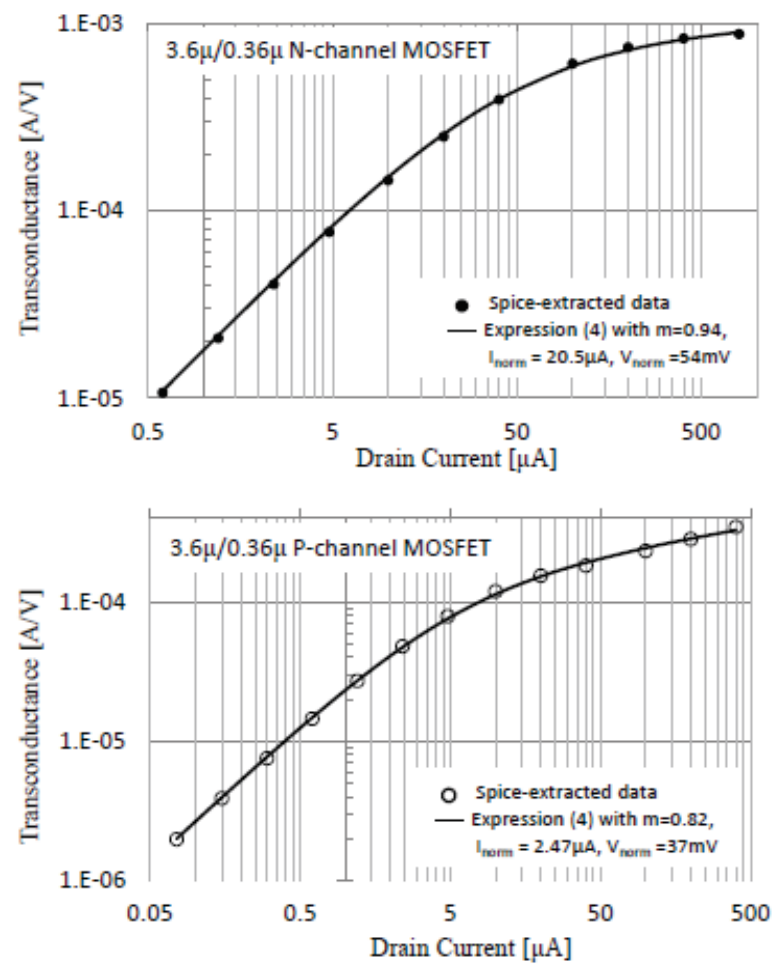

Figure 3. Spice-extracted gm-I data of an N-channel and a P-channel MOSFET from a representative $0.35 \mu \mathrm{m}$ process fitted using expression (4).
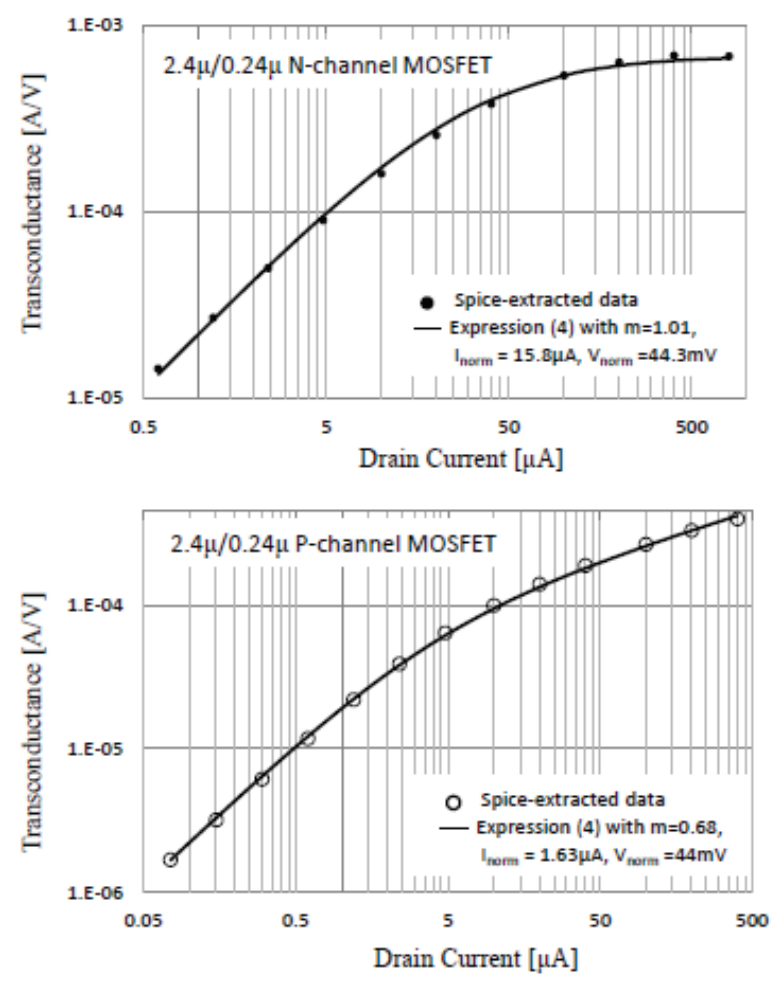

Figure 4. Spice-extracted gm-I data of an N-channel and a P-channel MOSFET from a representative $0.25 \mu \mathrm{m}$ process fitted using expression (4)
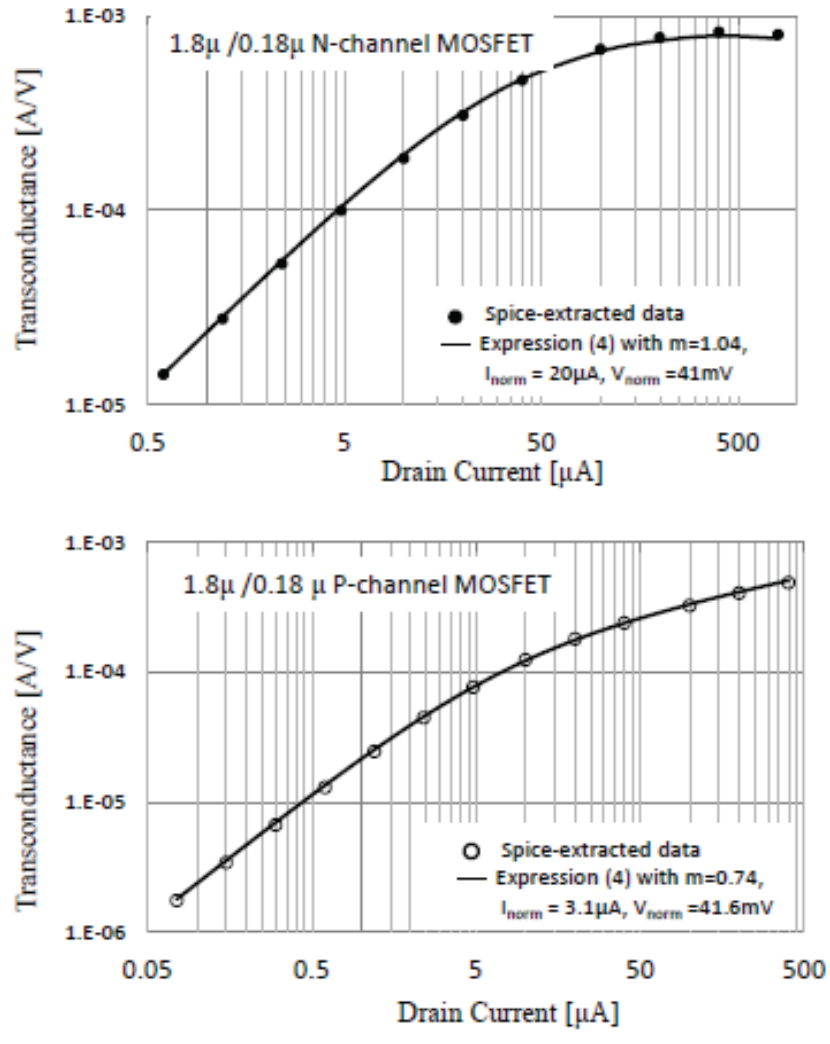

Figure 5. Spice-extracted gm-I data of an N-channel and a P-channel MOSFET from a representative $180 \mathrm{~nm}$ process fitted using expression (4).

TABLE I. FItTING PARAmeters AND MAXIMUM EROROR FOR SiX $\mathrm{N}$-CHANNEL MOSFETS. ALL W/L $=10$.

\begin{tabular}{|c|c|c|c|c|c|c|}
\hline \multirow{2}{*}{ Process } & \multirow{2}{*}{ Vendor } & \multirow{2}{*}{$\begin{array}{c}\text { Drawn } \\
\mathrm{L}_{\min }\end{array}$} & \multicolumn{3}{|c|}{ Fitting Parameters } & \multirow{2}{*}{$\begin{array}{l}\text { Max. } \\
\text { Error }\end{array}$} \\
\hline & & & $\mathrm{m}$ & $\mathrm{I}_{\text {norm }}$ & $V_{\text {norm }}$ & \\
\hline \multirow{2}{*}{$0.35 \mu \mathrm{m}$} & $\# 1$ & $0.4 \mu \mathrm{m}$ & 0.91 & $14.0 \mu \mathrm{A}$ & $52.0 \mathrm{mV}$ & $4.7 \%$ \\
\hline & $\# 2$ & $0.36 \mu \mathrm{m}$ & 0.94 & $20.5 \mu \mathrm{A}$ & $54.0 \mathrm{mV}$ & $4.2 \%$ \\
\hline \multirow{3}{*}{$0.25 \mu \mathrm{m}$} & $\# 1$ & $0.24 \mu \mathrm{m}$ & 0.87 & $9.9 \mu \mathrm{A}$ & $43.5 \mathrm{mV}$ & $6.0 \%$ \\
\hline & $\# 2$ & $0.24 \mu \mathrm{m}$ & 1.01 & $15.8 \mu \mathrm{A}$ & $44.3 \mathrm{mV}$ & $7.5 \%$ \\
\hline & $\# 1$ & $0.18 \mu \mathrm{m}$ & 1.04 & $20.0 \mu \mathrm{A}$ & $41.0 \mathrm{mV}$ & $4.7 \%$ \\
\hline $0.18 \mu \mathrm{m}$ & $\# 2$ & $0.18 \mu \mathrm{m}$ & 0.98 & $16.6 \mu \mathrm{A}$ & $43.9 \mathrm{mV}$ & $6.0 \%$ \\
\hline
\end{tabular}

TABLE II. FitTing PARAMETERS AND MAXIMUM ERRor FOR SiX P-CHANNEL MOSFETS. ALL W/L=10.

\begin{tabular}{|c|c|c|c|c|c|c|}
\hline \multirow{2}{*}{ Process } & \multirow{2}{*}{ Vendor } & \multirow{2}{*}{$\begin{array}{c}\text { Drawn } \\
\mathrm{L}_{\min }\end{array}$} & \multicolumn{3}{|c|}{ Fitting Parameters } & \multirow{2}{*}{$\begin{array}{l}\text { Max. } \\
\text { Error }\end{array}$} \\
\hline & & & $\mathrm{m}$ & $I_{\text {norm }}$ & $\mathrm{V}_{\text {norm }}$ & \\
\hline \multirow{2}{*}{$0.35 \mu \mathrm{m}$} & $\# 1$ & $0.4 \mu \mathrm{m}$ & 0.71 & $1.65 \mu \mathrm{A}$ & $44.5 \mathrm{mV}$ & $4.3 \%$ \\
\hline & $\# 2$ & $0.36 \mu \mathrm{m}$ & 0.82 & $2.47 \mu \mathrm{A}$ & $37.0 \mathrm{mV}$ & $5.2 \%$ \\
\hline \multirow{3}{*}{$0.25 \mu \mathrm{m}$} & $\# 1$ & $0.24 \mu \mathrm{m}$ & 0.70 & $1.81 \mu \mathrm{A}$ & 44.0 & $4.4 \%$ \\
\hline & $\# 2$ & $0.24 \mu \mathrm{m}$ & 0.68 & $1.63 \mu \mathrm{A}$ & $44.0 \mathrm{mV}$ & $5.2 \%$ \\
\hline & $\# 1$ & $0.18 \mu \mathrm{m}$ & 0.74 & $3.1 \mu \mathrm{A}$ & $41.0 \mathrm{mV}$ & $4.7 \%$ \\
\hline $0.18 \mu \mathrm{m}$ & $\# 2$ & $0.18 \mu \mathrm{m}$ & 0.77 & $2.82 \mu \mathrm{A}$ & $43.9 \mathrm{mV}$ & $6.0 \%$ \\
\hline
\end{tabular}




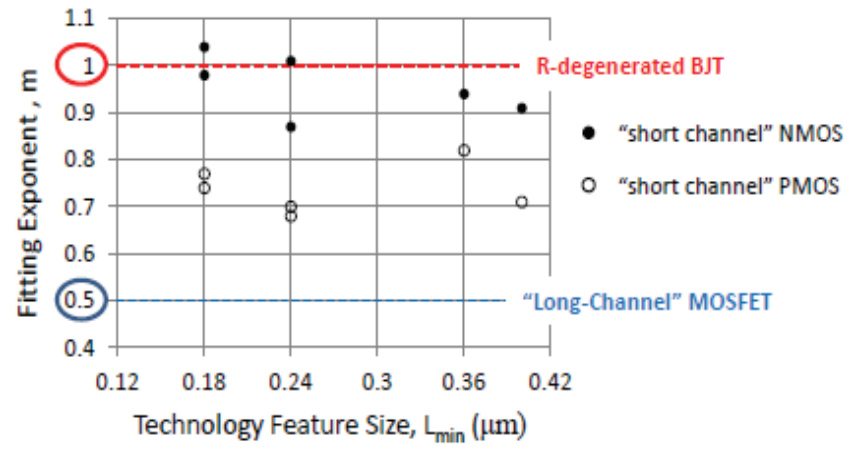

Figure 6. Exponents $m$ as a function of technology feature size and device type.

As shown in Table I, modeling the $g_{m}-I$ dependence of $\mathrm{N}$-channel devices (operated in strong inversion) requires exponents with values ranging from 0.9 to approximately 1.05. This implies that modern N-channel MOSFETs have $\mathrm{g}_{\mathrm{m}}$-I dependence that resembles the gm-I dependence of an emitter-degenerated BJT! The results presented in Table II demonstrate that modern P-channel MOSFETs are neither "long-channel" devices nor "degenerated BJTs". Their exponents range from 0.7 to 0.8 - nearly equidistant from 0.5 and 1.0. These observations are depicted graphically in Fig. 6.

\section{CONCLUSSIONS}

"Universal" $\mathrm{g}_{\mathrm{m}} \mathrm{I}$ expression is presented. The proposed model was shown capable of capturing the transconductancecurrent dependence of "long-channel" MOSFETs, "shortchannel" MOSFETs and BJTs with resistive emitter degeneration. The presented model was validated by curvefitting of gm-I data obtain from Spice simulations. Despite its non-physical nature, the $g_{m}-I$ model has been found invaluable for classroom instruction, by-hand design of analog circuits and comparison of CMOS technologies having different supply voltages and feature size.

\section{ACKNOWLEDGMENT}

The author would like to thank his former student Mr. Michael Spahn for collecting the Spice data used in this study.

\section{REFERENCES}

[1] P.R. Gray, P.J. Hurst, S.H. Lewis and R.G. Meyer, Analysis and Design of Analog Integrated Circuits, $4^{\text {th }}$ ed., John Wiley \& Sons, 2001, pp. $758-759$

[2] R. C.Jaeger and T. N. Blalock, Microelectronics Circuit Design, $4^{\text {th }}$ ed., McGraw Hill, 2011, pp. 817-818.

[3] F. Silveira, D. Flandre, and P. G. A. Jespers, "A gm/ID based methodology for the design of CMOS analog circuits and its application to the synthesis of a silicon-on-insulator micropower OTA," IEEE J. Solid-State Circuits, vol. 31, pp. 1314-1319, Sept. 1996.

[4] A. I. A. Cunha, M. C. Schneider, and C. Galup-Montoro, "An MOS transistor model for analog circuit design", IEEE J. Solid-State Circuits, vol. 33, no 10, pp. 1510-1519, October 1998.

[5] S. Yan and E. Sánchez-Sinencio, "Low voltage analog circuit design techniques: A tutorial," IEICE Trans. Fund., vol. E83, no. 2, pp. 1-17, Feb. 2000

[6] A. Girardi, and S. Bampi, "Power Constrained Design Optimization of Analog Circuits Based on Physical gm/ID Characteristics", Journal of Integrated Circuits and Systems, Vol. 2, No. 1, pp. 22-28, 2007.

[7] P. Jespers, The gm/ID Methodology, A Sizing Tool for Low-Voltage Analog Cmos Circuits, Springer, New York, NY, USA, 2009.

[8] O. C. F. Gouveia, A. I. A. Cunha, M. C. Schneider, and C. GalupMontoro. The ACM model for circuit simulation and equations for SMASH. [Online], available from http://www.dolphin.fr

[9] H. Shichman and D. A. Hodges. "Modeling and simulation of insulated-gate field-effect transistor switching circuits." IEEE J. Solid State Circuits, SC-3, pp. 285-289, Sept. 1968.

[10] MOSIS Fabrication Precesses, http://www.mosis.com/products/fabprocesses

[11] Wafer Wafer Electrical Test Data and SPICE Model Parameters, http://www.mosis.com/pages/Technical/Testdata/index 